\title{
Robust Information Security System Using Steganography, Orthogonal Code and Joint Transform Correlation
}

\author{
M. Nazrul Islam ${ }^{1}$, Muhammad Faysal Islam ${ }^{2}$ and Kamal Shahrabi ${ }^{3}$ \\ ${ }^{1}$ Department of Security Systems, State University of New York, Farmingdale, New York, USA; \\ E-mail: islamn@farmingdale.edu \\ ${ }^{2}$ Department of Engineering Management and Systems Engineering, George Washington \\ University, Washington, DC, USA; E-mail: mfi@gwmail.gwu.edu \\ ${ }^{3}$ Department of Electrical and Computer Engineering Technology, State University of New York, \\ Farmingdale, New York, USA; E-mail: Kamal.Shahrabi@farmingdale.edu
}

\begin{abstract}
The paper presents a novel and efficient information security technique by employing orthogonal coding scheme, encoded steganography and nonlinear encryption scheme involving joint transform correlation. Multiple biometric signatures are encoded using individual orthogonal codes and then multiplexed together. The encoded and multiplexed image is then embedded inside a cover image utilizing a new steganography technique. Here, a color cover image is first decomposed into three color components, red, green and blue, each of which is used to embed one set of biometric signatures. In each component image, one bit from the three least significant bits is selected using another secret key which is then replaced by the information bit. The component stego images are combined to form the color stego image, which is again encrypted using a multiple phase-shifted reference joint transform correlation (MRJTC) technique. The MRJTC technique is a nonlinear encryption process which increases the security strength significantly against any unauthorized access. The encoded steganography process reduces the vulnerability of an intruder retrieving any information through any steganalysis attack. In addition, the orthogonal encoding scheme enhances the robustness by making the biometric information almost inaccessible without authorization.
\end{abstract}

Index Terms: biometric signature, color image processing, orthogonal coding, joint transform correlation, steganography.

\section{INTRODUCTION}

With the growth of digital technology, the issues with the confidentiality and integrity of information are also growing at an alarming rate. Personal identification information requires robust security techniques to prevent from any unauthorized access. Biometric signatures, including fingerprints, iris, hand geometry, palm print, gestures, have proven to be efficient tools in establishing the identity of an individual because they contain unique characteristics of a human [1 - 4]. However, a great challenge with biometrics-based security systems is the variation and distortion of biometrics with time, place, and environment; additional limitations 
are non-cooperation of individuals, and preservation of biometric information [5 - 8]. Biometric information can be preserved and protected through steganography technique, where the least significant bit (LSB) of a cover image is replaced by the corresponding information bit [9, 10]. Traditional steganography techniques are vulnerable to steganalysis attacks where an intruder can try to retrieve the secret information by monitoring the LSBs of the cover image [11, 12]. A number of digital encryption techniques have also been proposed in the literature to protect biometric information [13-14]; however, they are typically linear processes, where there are always chances of decoding, completely or at least partly, the confidential information without knowing the secret keys.

The objective of this paper is to develop a novel and robust technique to protect biometric information employing orthogonal coding scheme, encoded steganography and nonlinear encryption process. Multiple biometric signatures are encoded and then multiplexed together in the form of a single image using orthogonal codes [15]. Then the encoded information is embedded into a color image, which is decomposed into three color channels, namely, red, green and blue, each of which is used to embed a set of biometric signatures. The proposed security system also employs a second set of keys to select one bit from the three LSBs of the cover image pixels for information hiding purpose [16-17]. Finally, the stego image is encrypted using a multiple phase-shifted reference joint transform correlation (MRJTC) technique, which incorporates a nonlinear transform-based encryption process [18]. The proposed information security system yields a high level of robustness against any security attacks by employing threefold encryption, namely, orthogonal coding, random bit replacement, and nonlinear encryption. In addition, the technique can accommodate a number of different biometric information in the same cover image while maintaining negligible amount of distortion when compared with original color cover image.

\section{PERFORMANCE ANALYSIS}

The overall block diagram of the proposed information security system is shown in Fig. 1. The input biometric signatures are first encoded using individual orthogonal codes and then multiplexed together. The encoded and multiplexed image is now embedded into the cover image by using a secret key for bit selection and replacement purpose. The stego image is finally encrypted using another set of keys employing the MRJTC technique.

The orthogonal encoding scheme is depicted in Fig. 2, where the input images containing biometric information are expanded in one dimension and then multiplied by the respective orthogonal code. The individual encoded images are then superimposed on a common spatial domain. If $g_{i}(x, y)$ is the expanded form of the $i$ 'th input image $f_{i}(x, y)$, and $k_{i}(x, y)$ is the respective orthogonal code, then the encoded and multiplexed image can be obtained as

$$
t(x, y)=\sum_{i=1}^{N} k_{i}(x, y) g_{i}(x, y)
$$

A Walsh code is employed in this paper to produce the orthogonal codes, which can be implemented by applying the Hadamard transform on 0 (zero) repeatedly as described below [19].

$$
H_{1}=[0]
$$




$$
H_{2 n}=\left\lfloor\begin{array}{ll}
H_{n} & H_{n} \\
H_{n} & \bar{H}_{n}
\end{array}\right\rfloor
$$

The set of codes are generated in the form of a square matrix, where the length of the matrix is $2^{m}$ and $m$ is the number of independent codes.

Then a color cover image is selected as the cover image to hide the confidential biometric information. The cover image can be any image, even another biometric image like face image. The color image is first split into its three color components, namely, red, green and blue, each of which is used to hide one set of biometric signatures. Figure 3 shows the encoded steganography technique for one color channel; the same scheme will be repeated for all the three color channels. The proposed steganography process employs a pseudo-random plane generated using a secret key to select a bit from the three LSBs of each pixel. The selected bits from the cover image are then replaced by the corresponding bits of the encoded and multiplexed image.

Figure 4 shows the block diagram of the nonlinear MRJTC-based encryption technique for securing the stego image. An encryption key is formed in the shape of an image having the same dimensions as the image to be encrypted but having random pixel values. The encryption key, $c(x, y)$, is fed to four parallel processing channels after phase shifting by $0^{\circ}, 90^{\circ}, 180^{\circ}$, and $270^{\circ}$, respectively. The input plaintext stego image, $d(x, y)$, is added to the phase-shifted keys in each channel, which yields four joint images as given by

$$
\begin{gathered}
f_{1}(x, y)=c(x, y)+d(x, y) \\
f_{2}(x, y)=j c(x, y)+d(x, y) \\
f_{3}(x, y)=-c(x, y)+d(x, y) \\
f_{4}(x, y)=-j c(x, y)+d(x, y)
\end{gathered}
$$

Applying Fourier transformation to each of the joint images in Eqs. (4)-(7), the magnitude spectra are recorded as four joint power spectrum (JPS) signals as given by

$$
\begin{gathered}
S_{1}(u, v)=\left|F_{1}(u, v)\right|^{2}=|C(u, v)|^{2}+|D(u, v)|^{2}+C(u, v) D^{*}(u, v)+C^{*}(u, v) D(u, v) \\
S_{2}(u, v)=\left|F_{2}(u, v)\right|^{2}=|C(u, v)|^{2}+|D(u, v)|^{2}+j C(u, v) D^{*}(u, v)+C^{*}(u, v) D(u, v) \\
S_{3}(u, v)=\left|F_{3}(u, v)\right|^{2}=|C(u, v)|^{2}+|D(u, v)|^{2}-C(u, v) D^{*}(u, v)-C^{*}(u, v) D(u, v) \\
S_{4}(u, v)=\left|F_{4}(u, v)\right|^{2}=|C(u, v)|^{2}+|D(u, v)|^{2}-j C(u, v) D^{*}(u, v)+j C^{*}(u, v) D(u, v)
\end{gathered}
$$

where $u$ and $v$ are the Fourier domain variables. The above JPS signals are again phase-shifted by $0^{\circ}, 90^{\circ}, 180^{\circ}$, and $270^{\circ}$, respectively. Then a modified JPS signal is developed using the following relation.

$$
S(u, v)=S_{1}(u, v)+j S_{2}(u, v)-S_{3}(u, v)-j S_{4}(u, v)=4 C^{*}(u, v) D(u, v)
$$

Inverse Fourier transformation of Eq. (12) yields the encrypted image as given by

$$
s(x, y)=4 c(x, y) \otimes d(x, y)
$$

where $\otimes$ denotes a convolution operation.

Now an authorized user having the correct set of keys can easily retrieve the original biometric information from the encrypted stego image of Eq. (13). As shown in Fig. 5, Fourier transformation is applied on the encrypted stego image and the result is multiplied by the 
encryption key. Then an inverse Fourier transformation can yield the decrypted image as given by

$$
e(x, y)=\mathfrak{J}^{-1}\{J(u, v) C(u, v)\}=4 t(x, y)
$$

As can be obvious from the above equation that the proposed technique can produce an uncorrupted form of the information by eliminating any unwanted signal due to correlation operation. To further enhance the decryption performance in order to remove any possible spurious signals, the signal in Eq. (14) is filtered by a fringe-adjusted filter (FAF) whose transfer function is given by

$$
H(u, v)=\frac{A(u, v)}{\left[B(u, v)+|C(u, v)|^{2}\right]}
$$

where the filter parameters, $A(u, v)$ and $B(u, v)$ can be adjusted to avoid having an optical gain greater than unity, and to overcome the pole problem, respectively.

The encrypted and multiplexed information can then be retrieved from the stego image following the technique depicted in Fig. 6. The user personal key is employed to recover the hidden image bits from the respective stego image pixel bits.

The recovered hidden image from the above step contains multiple biometric signatures that were encoded and multiplexed together. To decrypt specific information, the recovered image is multiplied with the respective encryption key. Then a threshold operation is performed depending on the format of the orthogonal code set used. If $k_{i}(x, y)$ represents the address code for the $i$ th biometric signature to be decrypted, then the output signal after the correlation operation is performed on the received signal can be expressed as

$$
r(x, y)=\iint_{\infty}\left[\sum_{k=1}^{N} K_{k}(u, v) T_{k}(u, u) \mid K_{i}(u-x, v-y) d u d v\right.
$$

where $K(u, v)$ and $T(u, v)$ are the Fourier transforms of the spatial domain signals, $k(x, y)$ and $t(x$, $y)$, respectively. It can be observed in Eq. (16) that only the desired signature will be produced at the output plane after the threshold operation, because other biometric signatures will not be able to contribute anything because of the fact that the cross-correlation between the any two orthogonal codes is always zero. Another salient feature of the proposed technique is that no additional processing step is required to demultiplex as this is automatically accomplished during the correlation operation.

\section{SIMULATION RESULTS}

The proposed information security system was simulated using MATLAB software in order to investigate its performance in various scenarios. Three sets of different biometric signatures were employed including fingerprints, iris scans, hand writing and text data. A Walsh code of length 4 was employed for encoding purpose.

Figures $8(\mathrm{a})-8(\mathrm{~d})$ show the sample binary biometric signatures to be encrypted in the red channel of the cover image using orthogonal code. The images were encoded using Walsh code and multiplexed together to form an encoded image as shown in Fig. 8(e), which contains the information from all the four input images of Figs. 8(a) - 8(d). 
Figures 9(a) - 9(d) show the second set of binary biometric signatures that were hidden in the green channel of cover image. The encoded and multiplexed image using orthogonal code is shown in Fig. 9(e), which contains all the four biometric information. Similarly, Fig. 10 shows the third set of biometric signatures that are encoded and multiplexed together in order to be embedded into the blue component of the cover image.

Figure 11 shows the effect of steganography process on a color cover image. The color image of Fig. 11(a) was used as the cover image to hide all the twelve biometric information depicted in Figs. $8-10$. The resultant stego image is shown in Fig. 11(b). It can be obvious by comparing these two images that there is no visible change in the given image even after embedding twelve biometric signatures.

The cover image was actually split into its color components, red, green, and blue. Each color component was used to contain the respective set of biometric signatures through the bit replacement process. Figures $12-14$ demonstrate the histogram analyses on the three color component images before and after steganography. As is obvious from the results that no significant change in the image characteristics has been observed.

Additional color images were employed as the cover images with varying background and contents. An example is depicted in Fig. 15 which has a significant amount of white background. However, the steganography process does not seem to have any visible effect on the image.

The stealth capability of the proposed technique was also investigated by using blind weighted stego image analysis to estimate the LSB change rates [21]. The simulation results are summarized in Table 1. Close change rate between a specific channel of the original cover image and resulted stego image indicates that it would be very difficult to determine if any information is hidden using a blind steganalysis method.

The stego image of Fig. 15(b) is then nonlinearly encrypted using the MRJTC technique, where the FAF parameters were chosen to as $A(u, v)=1.0$ and $B(u, v)=10^{-4}$. The image is placed in a joint input image along with the encryption key generated in the form of an image as shown in Fig. 16(a). The resultant encrypted stego image is shown in Fig. 16(e), which makes it obvious that the confidentiality of the input information is well-protected because of the nonlinear encryption process. Computer simulation experiment verified that the original stego can easily be recovered from the encrypted image of Fig. 16(b) without losing any information. Further investigations proved that the decryption process is so robust that it was still successful in retrieving the information under noisy environment and even when part of the image was occluded.

\section{CONCLUSION}

A novel and efficient information security system is proposed in this paper to protect biometric signatures from any unauthorized access. The technique employs orthogonal coding scheme to encode multiple biometric information and multiplex together which makes it almost impossible 
to decode even a part of the secret information without authorization. Though the simulation results presented in the paper included four biometric signatures, the orthogonal coding scheme can be extended to theoretically any number of information. The encoded biometric information is then embedded into a color cover image using a second set of keys to choose one bit from the three least significant bits. The steganography technique is so efficient in making the information completely hidden such that any steganalysis method can easily fail to retrieve any information. The stego image is finally encrypted using a modified joint transform correlation technique which offers a high level of security through incorporation of a nonlinear encryption process, however, involving a rather simple architecture. Simulation experiments verify that the proposed technique can yield an effective system for securing information, including biometric signatures, personal identification information, as well as confidential documents, and to verify the identity in real time.

\section{ACKNOWLEDGMENT}

This work is supported by a research grant from the Department of Energy, USA.

\section{REFERENCES}

[1] A. K. Jain, K. Nandakumar and A. Nagar, "Biometric template security," EURASIP Journal on Advances in Signal Processing, vol. 2008, ID. 579416, 2008.

[2] R. Sanchez-Reillo, C. Sanchez-Avila and A. Gonzalez-Marcos, "Biometric identification through hand geometry measurements," IEEE Transactions on Pattern Analysis and Machine Intelligence, vol. 22, no. 10, pp. 1168 - 1171, 2000.

[3] Y. C. Feng and P. C. Yuen, "Binary discriminant analysis for generating binary face template," IEEE Transactions on Information Forensics and Security, vol. 7, no. 2, pp. 613 $-624,2012$.

[4] M. Fouad, A. El Saddik, Z. Jiying and E. Petriu, "A fuzzy vault implementation for securing revocable iris templates," Proceedings on IEEE International Systems Conference (SysCon), pp. $491-494,2011$.

[5] L. Delac and M. Grgic, "A survey of biometric recognition methods," Proceedings of International Symposium on Electronics in Marine, pp. 184 - 193, 2004.

[6] D. Bala, "Biometrics and information security". Proceedings of the 5th annual conference on Information security curriculum development (InfoSecCD '08) ACM, pp. 64 - 66, 2008.

[7] J. E. Mills and S. Byun, "Cybercrimes against consumers: could biometric technology be the solution?," IEEE Internet Computing, vol. 10, no. 4, pp. 64 - 71, 2006.

[8] A. Chen and V. Chandran, "Biometric template security using higher order spectra," Proceedings of IEEE International Conference on Acoustics, Speech and Signal Processing, pp. $1730-1733,2010$.

[9] A. Cheddad, J. Condell, K. Curran and P. McKevitt, "Biometric inspired digital image steganography," Proceedings of 15th Annual IEEE International Conference and Workshop on the Engineering of Computer Based Systems (ECBS 2008), pp. 159 - 168, 2008.

[10] X. Luo, Z. Hu, C. Yang and S. Ga, "A secure LSB steganography system defeating sample pair analysis based on chaos system and dynamic compensation," Proceedings of The 8th 
International Conference on Advanced Communication Technology, vol. 2, pp. 1014 1019, 2006.

[11] A. D. Ker, "Steganalysis of embedding in two least-significant bits," IEEE Transactions on Information Forensics and Security, vol. 2, no. 1, pp. 46 - 54, 2007.

[12] M. Goljan, J. Fridrich and T. Holotyak, "New blind steganalysis and its implications," Proceedings of SPIE in Security, Steganography and Watermarking of Multimedia Contents VIII, vol. 6072, pp. 1 - 13, 2006.

[13] F. Pernus, S. Kovacic and L. Gyergyek, "Minutaie based fingerprint registration," IEEE Pattern Recognition, pp 1380, 1980.

[14] P. M. Mudegaonkar and R. P. Adgaonkar, "A novel approach to fingerprint identification using gabor filter-bank," ACEEE International Journal on Network Security, vol. 2, no. 3, pp. $10-14,2011$.

[15] M. N. Islam, "Encryption and multiplexing of fingerprints for enhanced security," Proceedings of IEEE Long Island Systems, Applications and Technology Conference (LISAT), 2011.

[16] M. F. Islam and M. N. Islam, "A secure approach for encrypting and compressing biometric information employing orthogonal code and steganography," SPIE Proceedings in Optical Pattern Recognition XXIII, 2012.

[17] M. F. Islam and M. N. Islam, "Efficient and robust information security system employing color encoded steganography and orthogonal encryption," Proceedings of IASTED International Conference on Visualization, Imaging and Image Processing, July 2012.

[18] M. N. Islam, "Color image encryption using multiple reference joint transform correlation," Proceedings of IEEE Long Island Systems, Applications and Technology Conference (LISAT), 2012.

[19] F. R. K. Chung, J. A. Salehi and V. K. Wei, "Optical orthogonal codes: design, analysis and applications," IEEE Transactions on Information Theory, vol. IT-35, pp. 595 - 604, 1989.

[20] R. McEvoy, J. Curran, P. Cotter and C. Murphy, "Fortuna: cryptographically secure pseudorandom number generation in software and hardware," Proceedings of Irish Signals and Systems Conference (IET), pp. 457 - 462, 2006.

[21] A. D. Ker and R. Böhme, "Revisiting weighted stego-image steganalysis," Proceedings of SPIE in Security, Forensics, Steganography, and Watermarking of Multimedia Contents X, vol. 6819, San Jose, CA, pp. 0501 - 0517, 2008. 


\title{
Robust Information Security System Using Steganography, Orthogonal Code and Joint Transform Correlation
}

\author{
M. Nazrul Islam ${ }^{1}$, Muhammad Faysal Islam ${ }^{2}$ and Kamal Shahrabi ${ }^{3}$
}

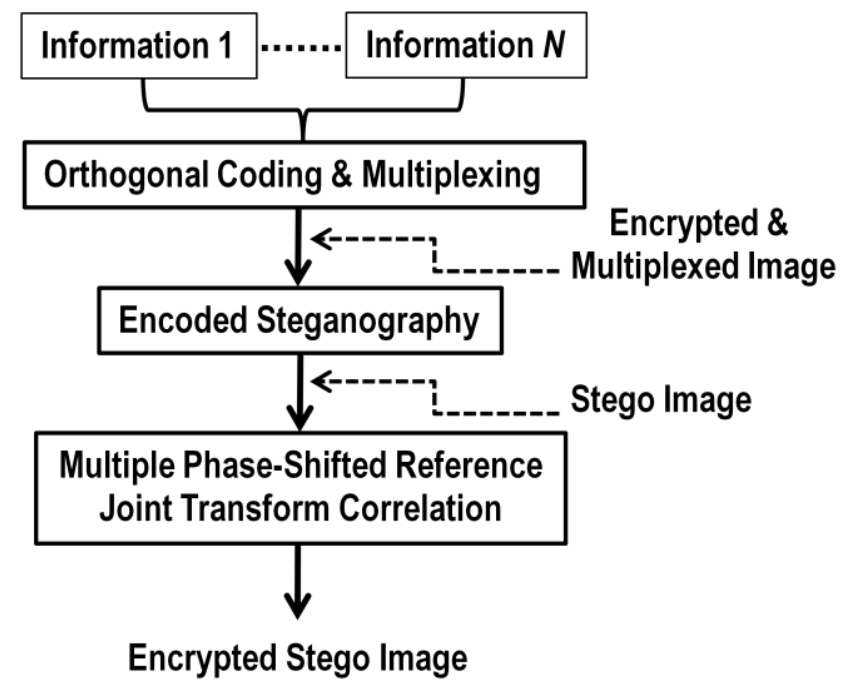

Figure 1: Block diagram of the proposed security system

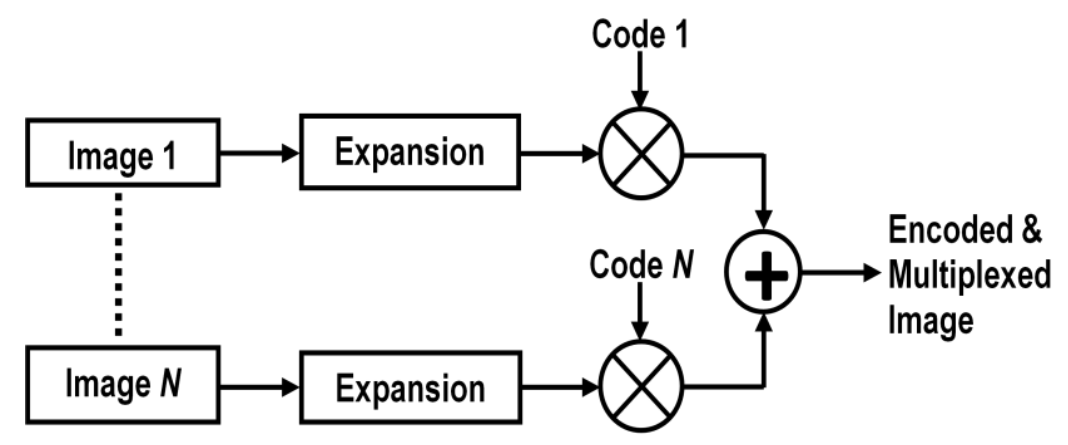

Figure 2: Block diagram of the orthogonal encoding technique 


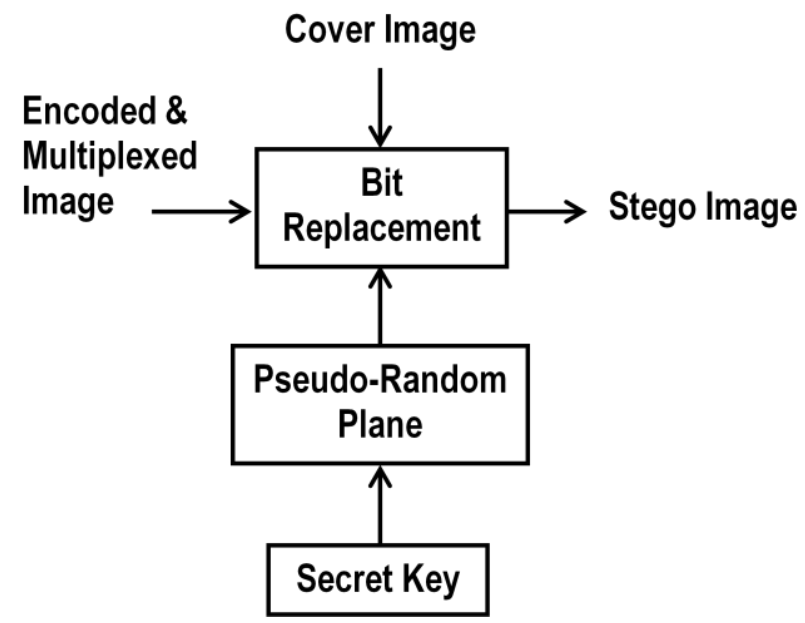

Figure 3: Block diagram of the encoded steganography technique

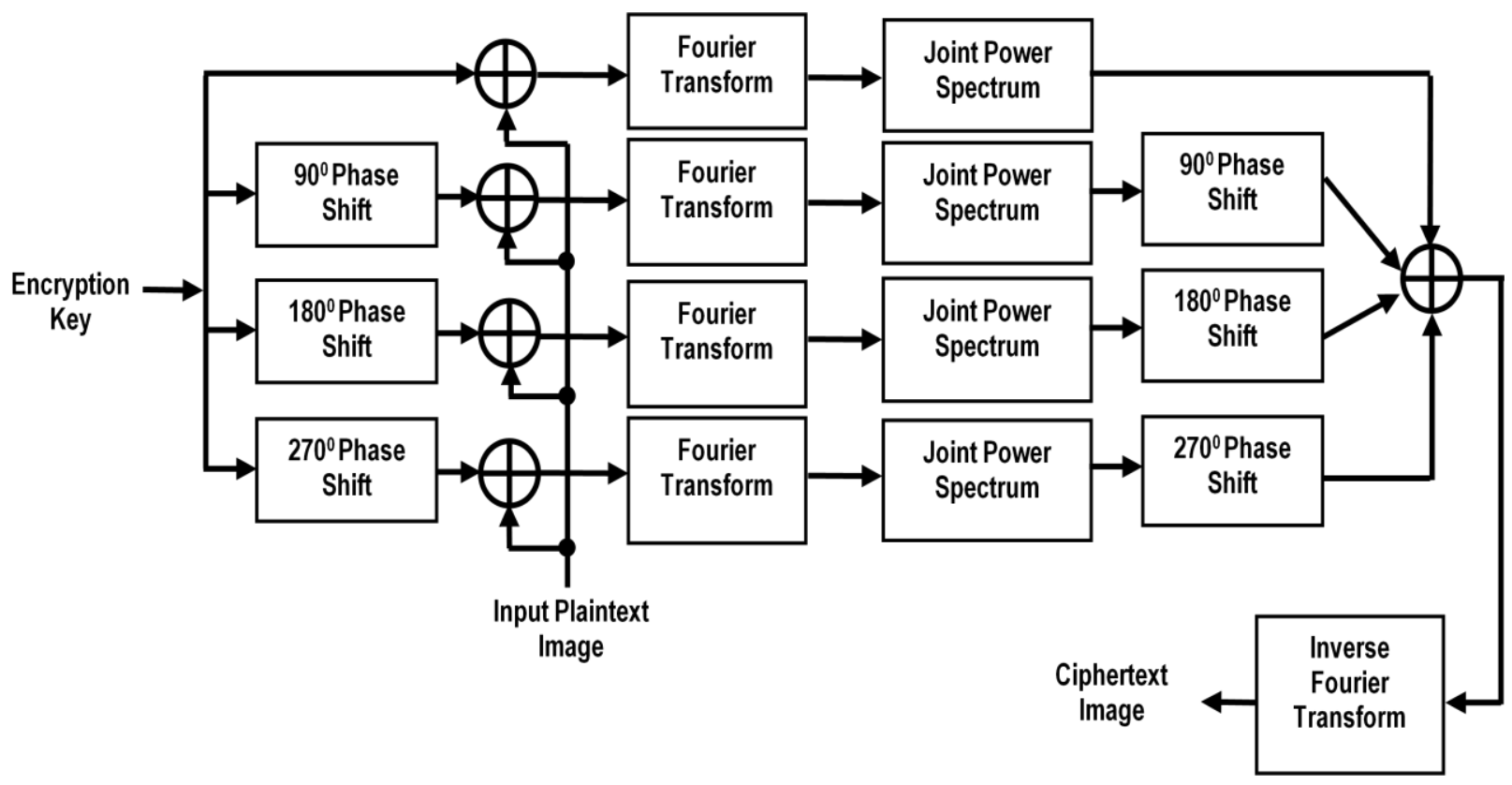

Figure 4: Block diagram of the MRJTC encryption technique

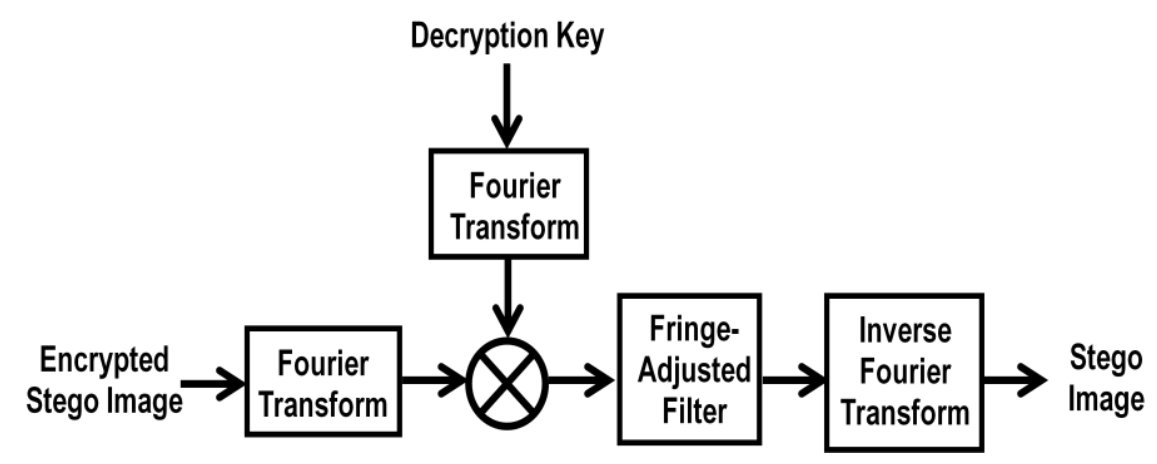


Figure 5: Block diagram of the MRJTC-based decryption technique

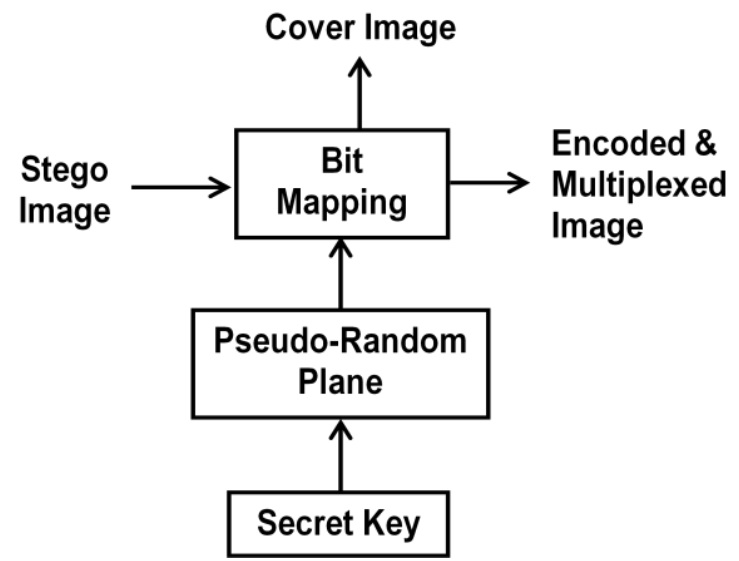

Figure 6: Block diagram of the bit mapping technique

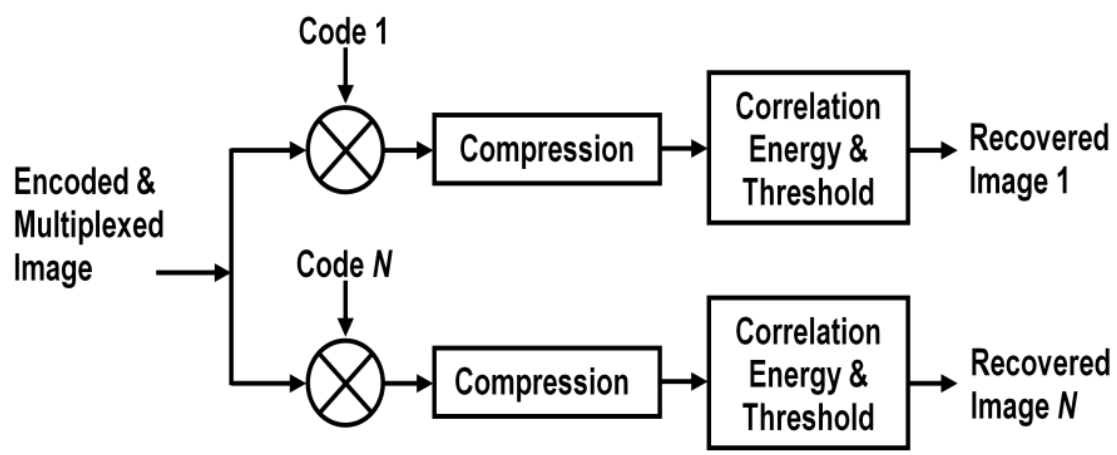

Figure 7: Block diagram of the orthogonal decoding technique

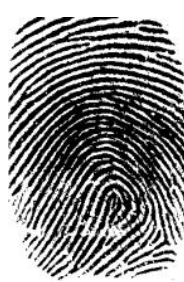

(a)

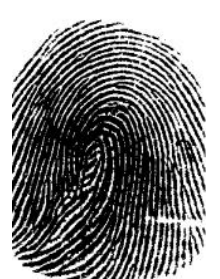

(b)

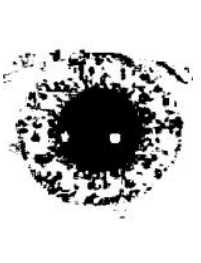

(c)

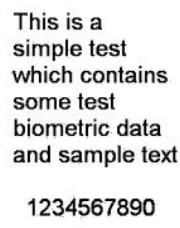

This is a

simple test

which contains

some test

biometric data

and sample text

1234567890

(d)

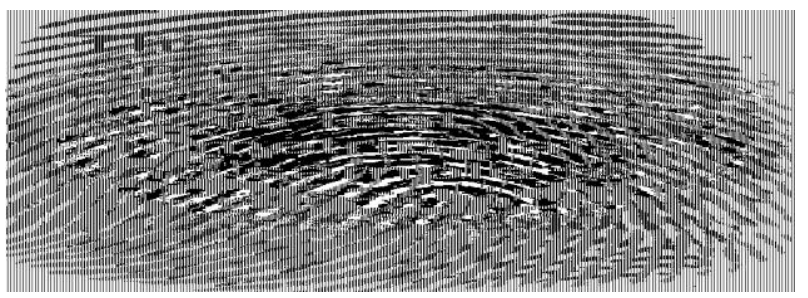

(e) 
Figure 8: Orthogonal coding and multiplexing of biometric signatures for red cover image: (a) - (d) input biometric information to be encrypted, (e) encrypted and multiplexed image

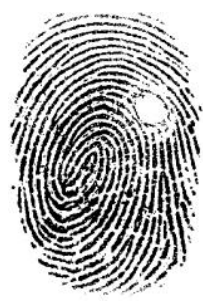

(a)

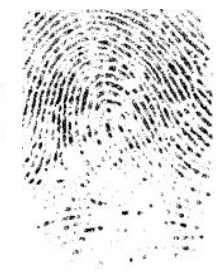

(b)

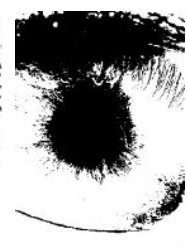

(c)
$1 \mathrm{H}$

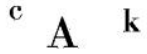

Random

Text

(d)

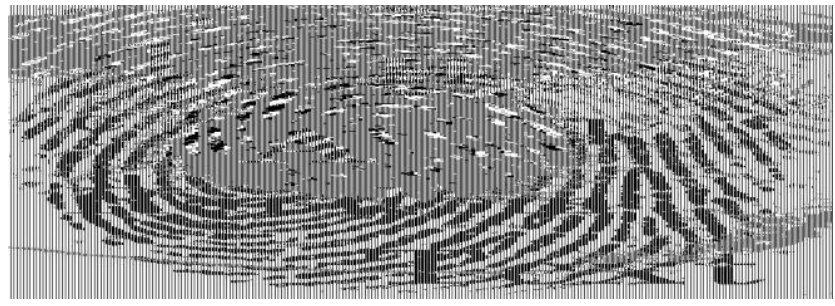

(e)

Figure 9: Orthogonal coding and multiplexing of biometric signatures for green cover image: (a) - (d) input biometric information to be encrypted, (e) encrypted and multiplexed image

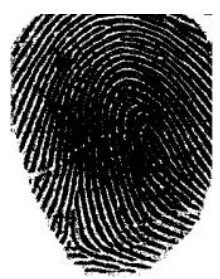

(a)

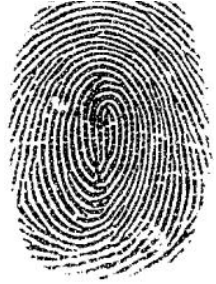

(b)

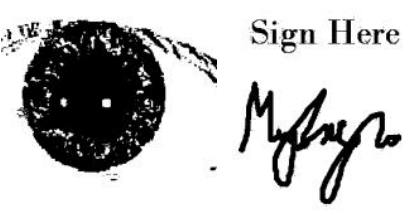

(c)

(d)

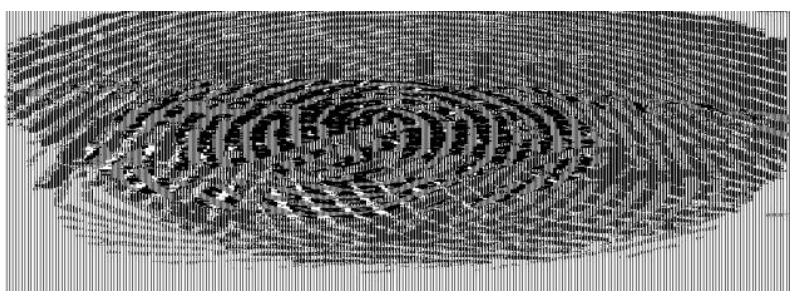

(e)

Figure 10: Orthogonal coding and multiplexing of biometric signatures for blue cover image: (a) - (d) input biometric information to be encrypted, (e) encrypted and multiplexed image 


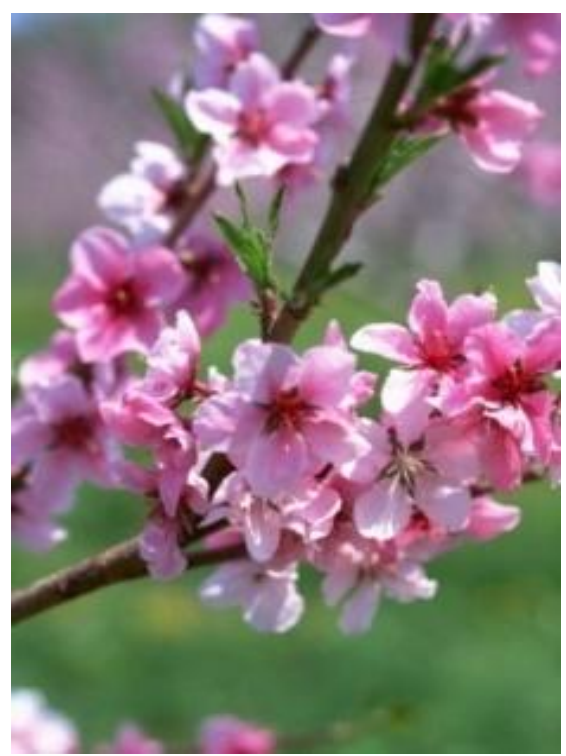

(a)

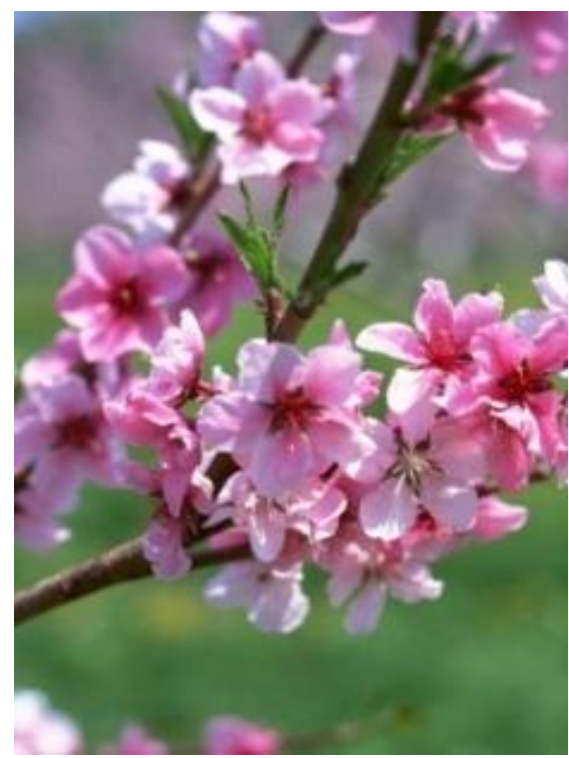

(b)

Figure 11: Effect of steganography on a color cover image: (a) image before steganography, and (b) image after embedding twelve different binary biometric signature images

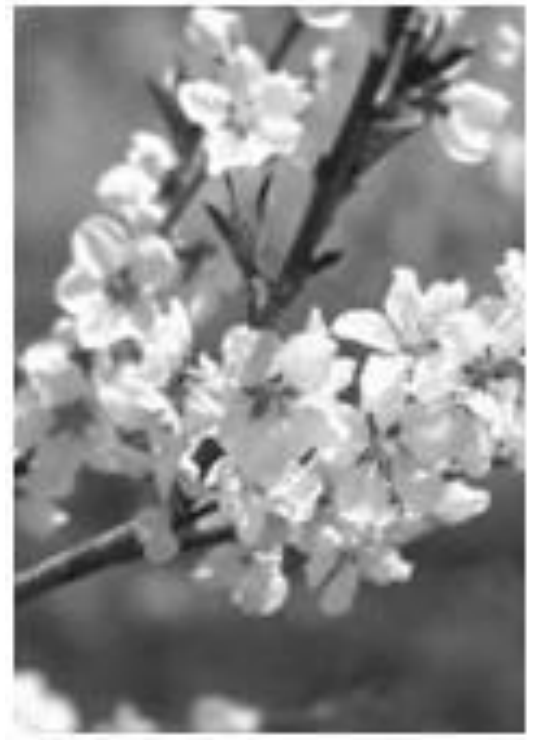

(a)

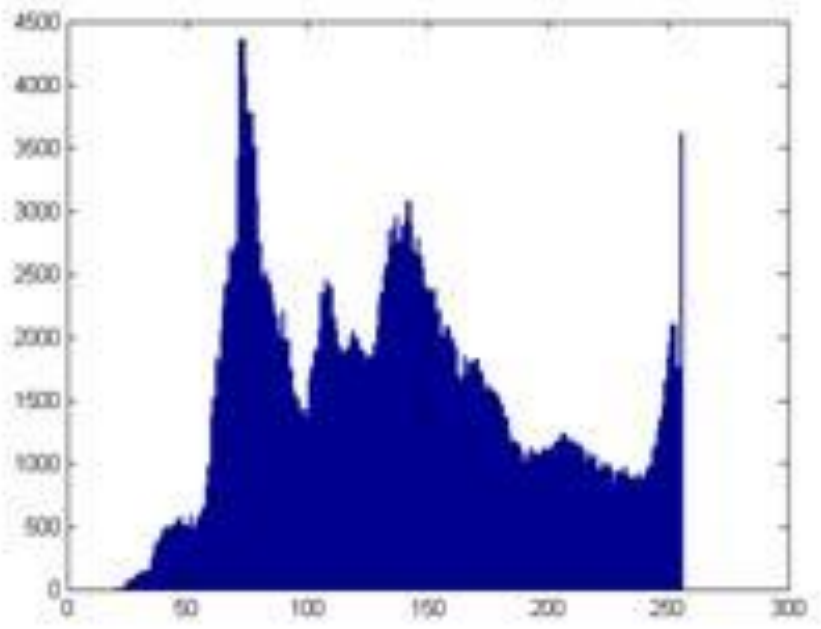

(b) 


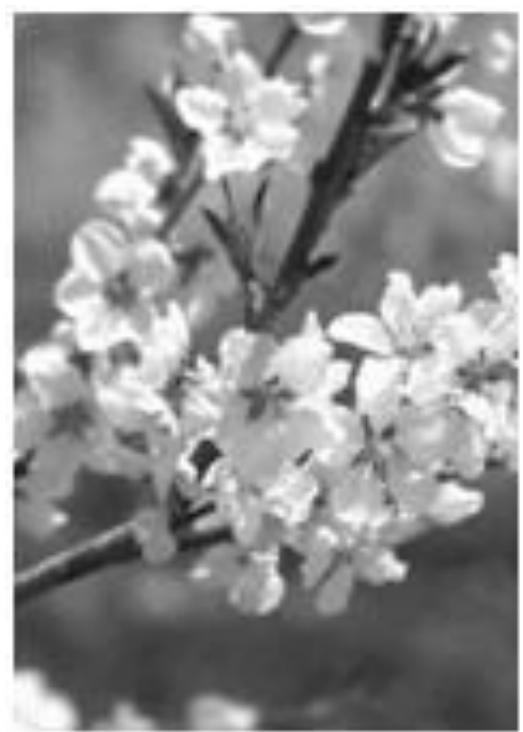

(c)

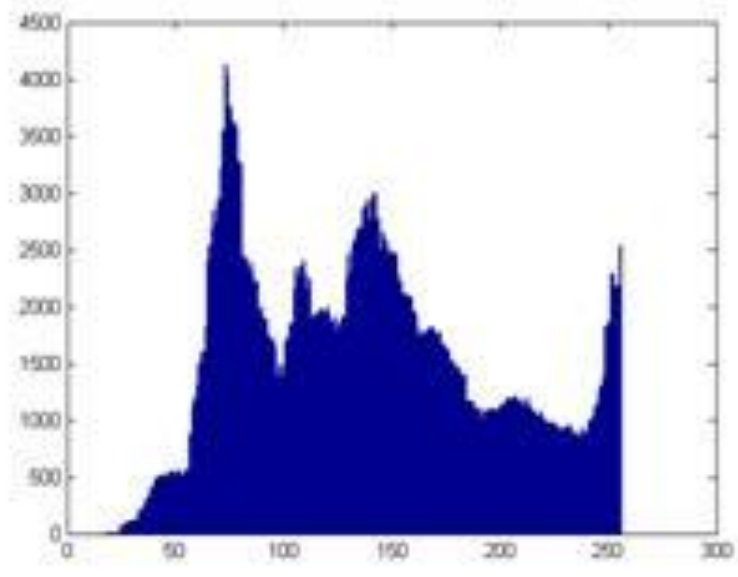

(d)

Figure 12: Effect of steganography on the red cover image: (a) image before steganography, (b) histogram of image in (a), (c) image after steganography, and (d) histogram of image in (c)

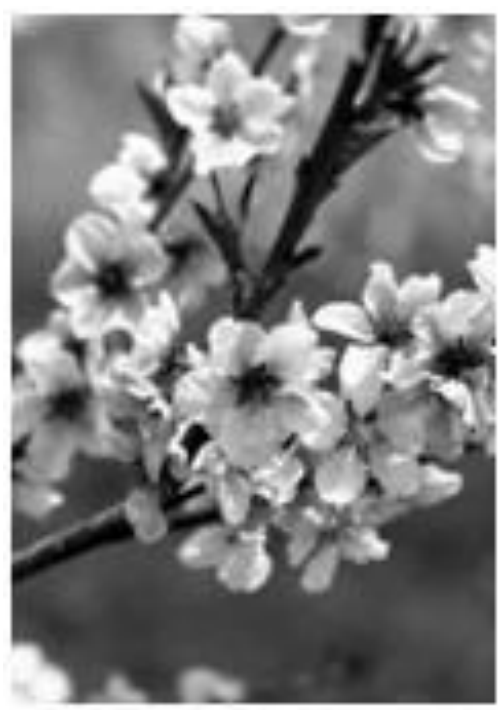

(a)

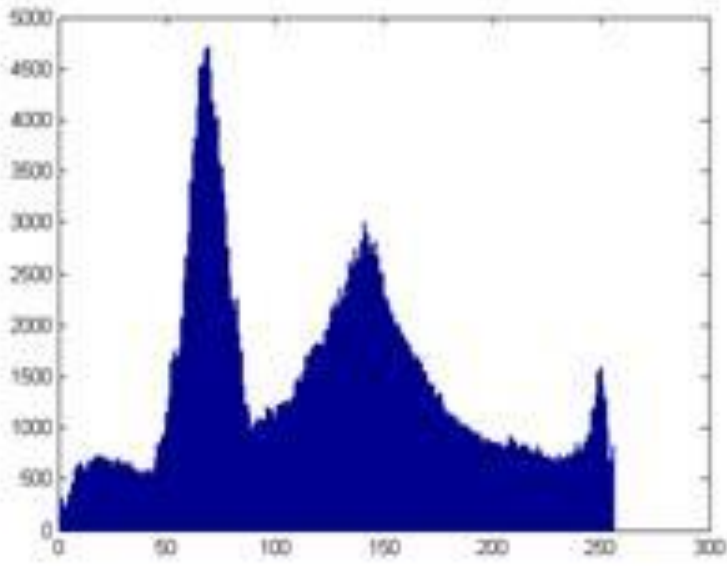

(b) 


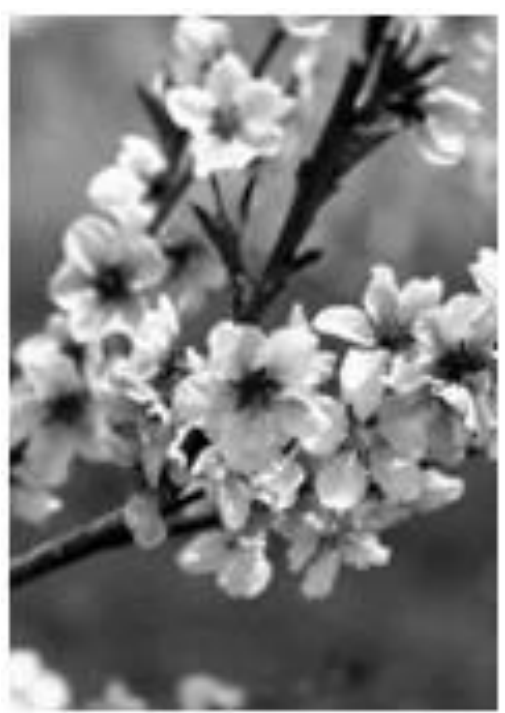

(c)

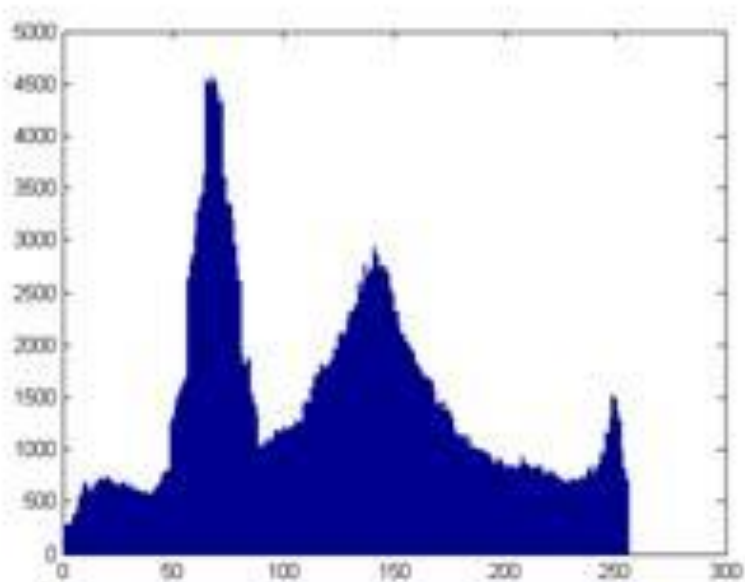

(d)

Figure 13: Effect of steganography on the green cover image: (a) image before steganography, (b) histogram of image in (a), (c) image after steganography, and (d) histogram of image in (c)

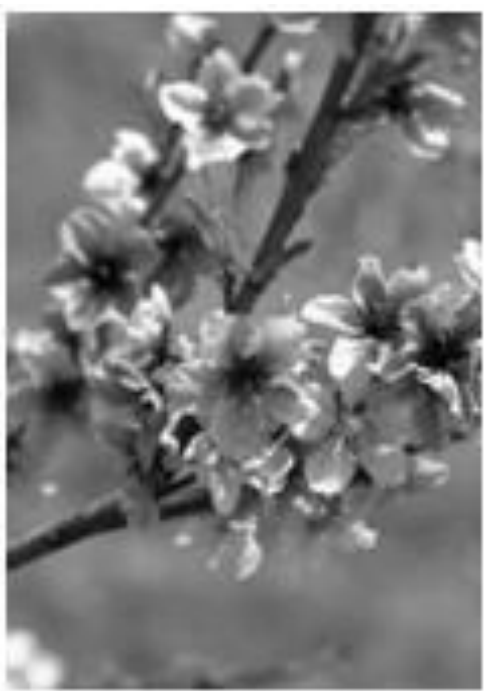

(a)

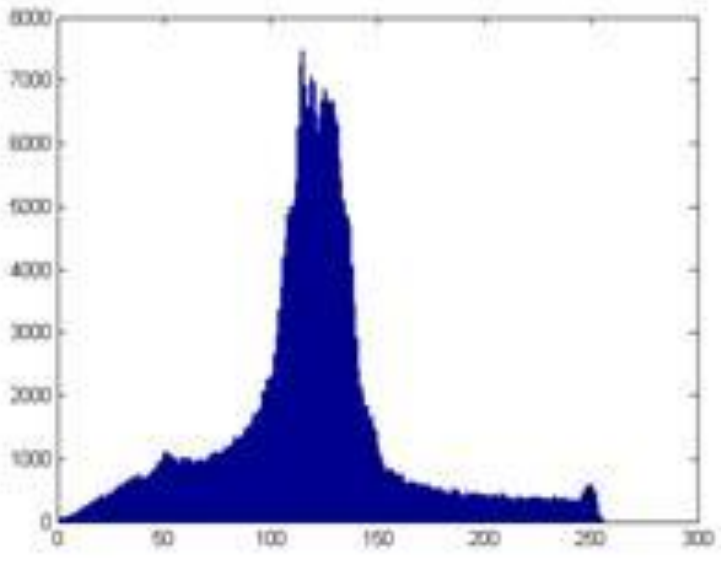

(b) 


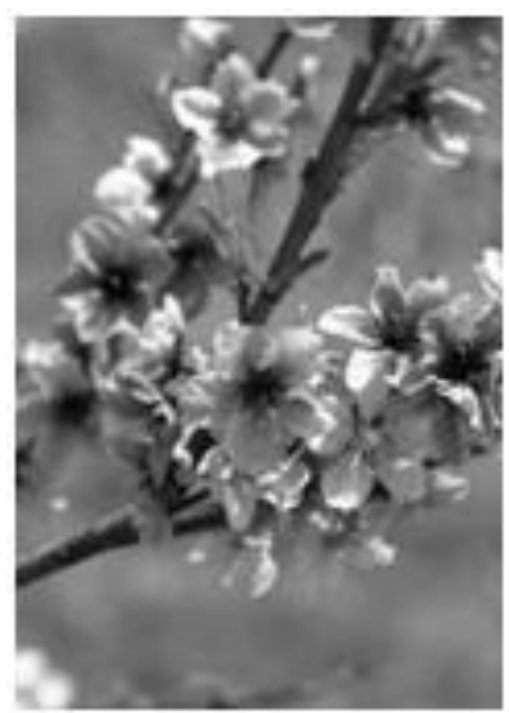

(c)

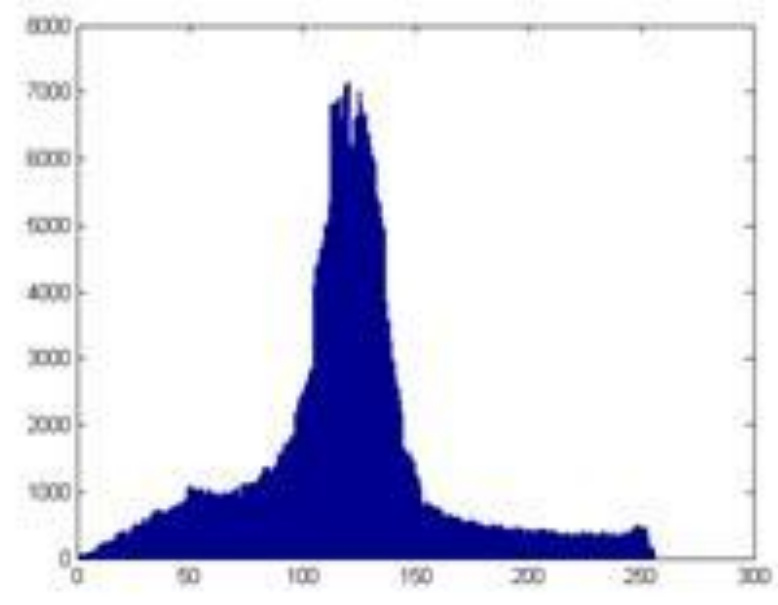

(d)

Figure 14: Effect of steganography on the blue cover image: (a) image before steganography, (b) histogram of image in (a), (c) image after steganography, and (d) histogram of image in (c)

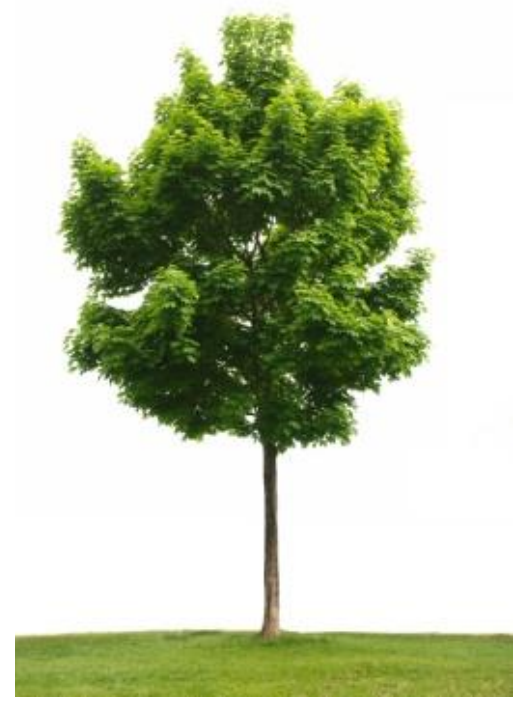

(a)

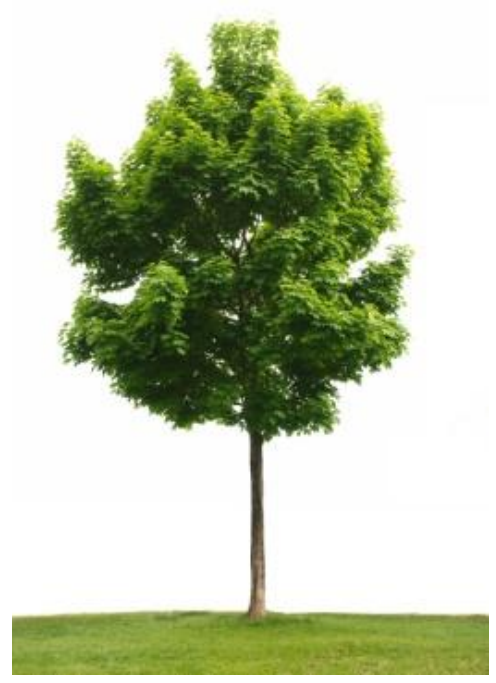

(b)

Figure 15: Effect of steganography on a color cover image: (a) image before steganography, and (b) image after embedding twelve different binary biometric signature images 


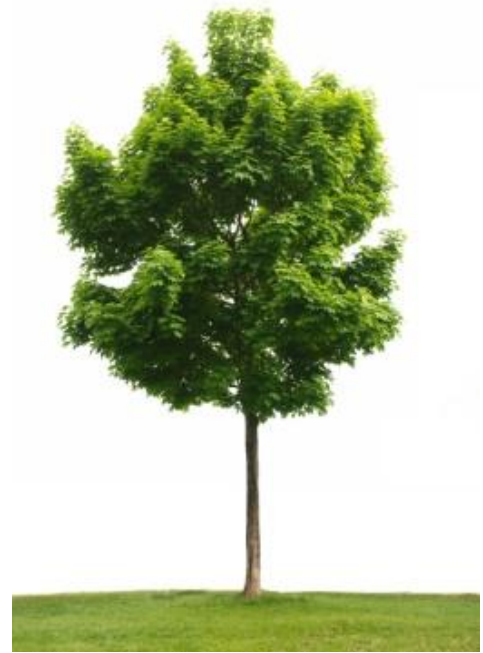

(a)

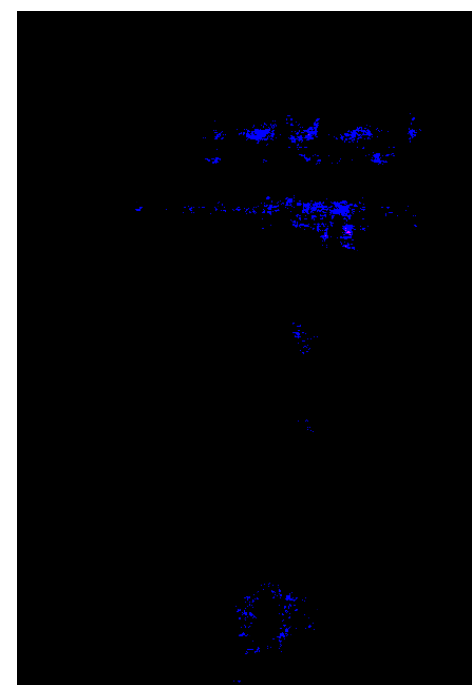

(b)

Figure 16: MRJTC-based encryption technique: (a) stego image, and (b) encrypted stego image 


\title{
Robust Information Security System Using Steganography, Orthogonal Code and Joint Transform Correlation
}

\author{
M. Nazrul Islam ${ }^{1}$, Muhammad Faysal Islam ${ }^{2}$ and Kamal Shahrabi ${ }^{3}$
}

Table 1: Weighted stego image change rates for original cover image and stego image

\begin{tabular}{|l|c|c|}
\hline \multicolumn{1}{|c|}{ Weighted Stego } & $\begin{array}{c}\text { Original } \\
\text { Cover } \\
\text { Image }\end{array}$ & $\begin{array}{c}\text { After } \\
\text { Steganography }\end{array}$ \\
\hline $\begin{array}{l}\text { Cover Image 1 } \\
\text { Red }\end{array}$ & 0.00069297 & 0.00071582 \\
\hline $\begin{array}{l}\text { Cover Image 1 } \\
\text { Green }\end{array}$ & 0.00138481 & 0.00140686 \\
\hline $\begin{array}{l}\text { Cover Image 1 } \\
\text { Blue }\end{array}$ & 0.00017203 & 0.00019150 \\
\hline $\begin{array}{l}\text { Cover Image 2 } \\
\text { Red }\end{array}$ & 0.00131138 & 0.00122920 \\
\hline $\begin{array}{l}\text { Cover Image 2 } \\
\text { Green }\end{array}$ & 0.00167064 & 0.00152484 \\
\hline $\begin{array}{l}\text { Cover Image 2 } \\
\text { Blue }\end{array}$ & 0.00094766 & 0.00114984 \\
\hline $\begin{array}{l}\text { Cover Image 3 } \\
\text { Red }\end{array}$ & 0.00919333 & 0.00838849 \\
\hline $\begin{array}{l}\text { Cover Image 3 } \\
\text { Green }\end{array}$ & 0.00329215 & 0.00334655 \\
\hline $\begin{array}{l}\text { Cover Image 3 } \\
\text { Blue }\end{array}$ & 0.00528524 & 0.00569346 \\
\hline $\begin{array}{l}\text { Cover Image 4 } \\
\text { Red }\end{array}$ & 0.04288447 & 0.04337175 \\
\hline $\begin{array}{l}\text { Cover Image 4 } \\
\text { Green }\end{array}$ & 0.08928525 & 0.09171086 \\
\hline $\begin{array}{l}\text { Cover Image 4 } \\
\text { Blue }\end{array}$ & 0.14010596 & 0.15743530 \\
\hline
\end{tabular}

